ESAIM: PROCEEDINGS, October 2011, Vol. 33, p. 1-9

C. Dobrzynski, T. Colin \& R. Abgrall, Editors

\title{
THE ROLE OF MECHANICS IN TUMOR GROWTH: MODELLING AND SIMULATION
}

\author{
D. Ambrosi ${ }^{1}$
}

\begin{abstract}
A number of biological phenomena are interlaced with classical mechanics. In this review are illustrated two examples from tumor growth, namely the formation of primordial networks of vessels (vasculogenesis) and the avascular phase of solid tumors. In both cases the formalism of continuum mechanics, accompanied by accurate numerical simulations, are able to shed light on biological controversies. The converse is also true: non-standard mechanical problems suggest new challenging mathematical questions.
\end{abstract}

\section{INTRODUCTION}

Mechanobiology is an area of increasing interest in continuum mechanics. The impressive improvements of experimental techniques offer to scientists a huge number of data, at several spatial scales. The accuracy of experimental measures and the novelty of the observed phenomena make the mathematical modelling of biological systems a very attractive field. Biochemistry currently plays a major role, but there is quite a consensus that mechanics, and in particular continuum mechanics, is the correct tool for an insight of several open questions, as, for instance, mechanotrasduction [17].

In this paper are sketched two examples of biological problems where the mathematical formalization has been an effective tool to understand the inner mechanisms of a biological system: solid tumor growth and vasculogenesis. The choice of the subjects and the way to treat them is manifestly driven by my own scientific activity and my personal taste. Both problems however share one common feature: the mathematical modelling and the numerical simulation is not purely addressed to reproduce numerically something observed in nature, but there is a more ambitious aim, or even two.

- It happens that the biological literature is controversial, as there are different possible explanations for one complex biological behavior. Sometimes several explanations look equally satisfactory at a qualitative level; the formalization of the phenomenology into equations that obey the laws of physics and their numerical solution represents an effective tool to discriminate quantitatively among possible mechanisms.

- The mathematical model of some biological systems are known and assessed, at least in simple geometries or with deterministically known parameters. Then numerics becomes the essential tool to face complex geometries or to account for the stochastic nature of the system. This application of mechanobiology becomes particularly important in medical applications, where predictions of the development of human diseases always face uncertain and patient-specific data.

In this review I will not go through the details of the biological behavior and mathematical modelling of the specific problems; the intent herein is to stress the original perspective that mathematics can provide in biology.

${ }^{1}$ MOX-Dipartimento di Matematica, Politecnico di Milano, 20133 Milano, Italy

(C) EDP Sciences, SMAI 2011 
ESAIM: PROCEEDINGS

\section{SOlid TUMOR GROWTH}

After Folkman \& Hochbergand [7], the typical experimental setting to determine the uncontrolled duplication rate of tumor cells in vitro is a cluster of cells, freely floating in a culture medium, called multicell spheroid. A tumor spheroid is therefore an ensemble of cells freely proliferating in an environment with large availability of nutrient. In particular, they have lost the ability to self-regulate their own number through a normal apoptosis mechanism. In absence of external cues the shape of the cell cluster is basically spherical, as gravity exerts no influence.

In the standard free-growth case, a plot of the diameter of the tumor vs. time typically exhibits an early stage of exponential growth, followed by a linear one. The transition from one regime to the other is mainly regulated by the availability of nutrient, that occurs by diffusion. In fact, when the size of the tumor $\bar{r}(t)$ is smaller than the typical diffusion length, the nutrient is everywhere available in the spheroid and the growth is volumetric:

$$
\frac{d \bar{r}^{3}}{d t} \simeq \bar{r}^{3}
$$

so that $\bar{r}(t) \simeq e^{t}$. Conversely, when the diameter of the spheroid is much larger than the penetration length, one obtains surface growth, that is

$$
\frac{d \bar{r}^{3}}{d t} \simeq \bar{r}^{2}
$$

and $\bar{r}(t) \simeq t$. See [4] and the references therein for a review of the literature on growth of spatially uniform models of avascular tumors.

The availability of nutrients is definitely the major factor affecting tumor growth, but other external agents can affect it. The mechanical influence of external loading on tumor growth has been investigated by Helmlinger et al. [11]. They design an experimental setup in order to control the traction applied at the boundary of clusters of cells grown in vitro, and check the influence of such a stress on the growth rate of the multicell spheroid.

In a standard apparatus, multicell spheroids freely grow floating in culture medium. The novelty of the work of these authors is to plug the tumor cells in a gel, produced at given stiffness by tuning suitably the solid phase concentration. As the spheroid grows, it displaces the surrounding gel, which then exerts a pressure at the surface of the tumor spheroid. An a priori mechanical characterization of the gel allows to calculate the pressure exerted by the gel on the spheroid depending on its radius.

The main result of these experiments is that the stress field reduces the final dimension of the spheroid. This behavior has been qualitatively reproduced by Ambrosi and Mollica [3] assuming that the spheroid is an hyperelastic material.

The assumption to model an aggregate of cells as an hyperelastic material simplifies a lot the analysis, but the mechanical assumption is too crude and later work aims to refine the theory considering the plastic properties of rearrangement between cells [2]. Stress relaxation can be introduced at a macroscopic level on the basis of cellular arguments. It is known that cells adhere each other via cadherin junctions. In standard experiments to test the adhesive strength of a cell, a microsphere is fixed to the tip of an atomic force microscopy cantilever. The microsphere is posed in contact with the cell and then the cantilever is slowly pulled away, at a constant speed. Typical experimental plots of force vs. displacement are shown in Fig. 1; the characteristic strength of a bond can be measured as a function of the displacement.

If a cluster of cells is subject to a sufficiently high tension, locally some bonds break and new ones form. At a macroscopic level, this argument suggests the introduction of a yield stress and the use of the plastic deformations formalism of continuum mechanics. In particular, the mechanism of cell attachment-detachment can be relevant during growth under an external load, when duplicating cells displace their neighbours only if the needed energy is available. The aim is therefore to include elasto-visco-plastic effects in the mechanics of cell aggregates. The starting point of the theory is the following experimental evidence, for cell aggregates under compression

i for a moderate amount of stress, the cell aggregate deforms elastically; 


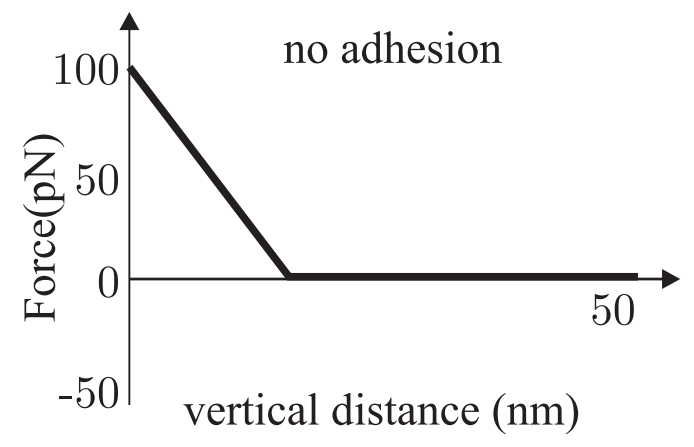

(a)

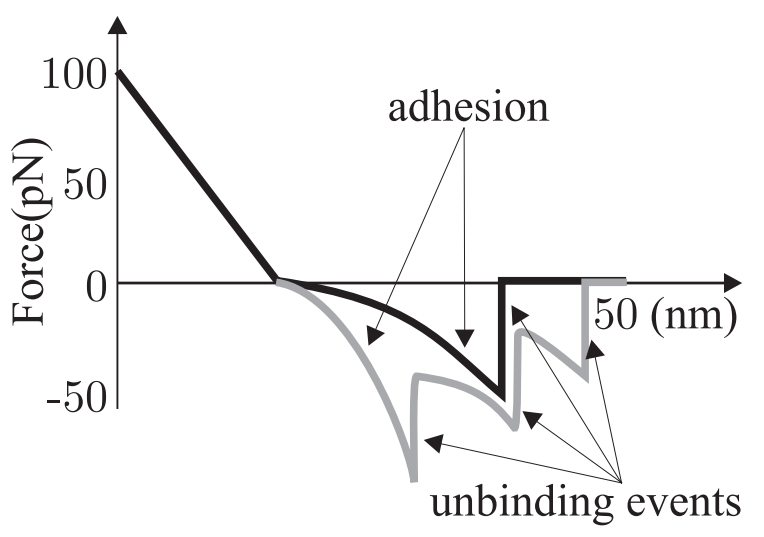

(b)

Figure 1. Typical plot of force vs. displacement produced by an adhesive bead attached to the surface of a cell. The initial behavior is elastic non-linear, but for larger displacements (and larger strains of the cell membrane) bonds start breaking with single or multiple unbinding events, until complete detachment. In this plot positive force indicates compression.

ii above a yield value the cell aggregate undergoes internal re-organization which is modelled at a macroscopic level as a visco-plastic deformation.

The formalization of these intuitive arguments from a cellular to a continuum level can be approached in terms of the plasticity theory: an example of visco-elastoplastic model of tumor growth is illustrated in [2]. Here below are resumed the main results of such a work in a slightly simplified way.

A useful paradigm for elastoplastic deformations is the multiplicative decomposition of the deformation gradient $\mathbf{F}$. When loading a solid material at large strains, it can happen that energy is provided externally in terms of work of external forces, without apparent displacement. This macroscopic behavior has a microscopic explanation: energy is not stored as strain energy, but is spent in form of internal rearrangement of the material that does not produce apparent macroscopic deformation. The basic idea is to represent such a physical behavior by the introduction of a virtual, intermediate configuration between the reference (relaxed) configuration and the current one. In such an intermediate, possibly evolving configuration, the body is stress free but, because of possible plastic rearrangements, it may not to coincide with the original relaxed configuration. The relaxed state can differ from the originally relaxed one because, during the deformation, cells in the configuration can undergo internal re-organization, which implies re-arranging of the adhesion links among the cells. We identify the deformation that takes from the current (loaded) state to the relaxed one without cell re-organization $\mathbf{F}_{e}$. The deformation gradient is then split as

$$
\mathbf{F}=\mathbf{F}_{e} \mathbf{F}_{p}
$$

The definition of the following tensors will turn useful in the next:

$$
\mathbf{B}_{e}=\mathbf{F}_{e} \mathbf{F}_{e}^{T}, \quad \mathbf{L}=\dot{\mathbf{F}} \mathbf{F}^{-1}
$$

We denote by $\tau$ the yield stress, the minimum tension that induces shearing. It is to be compared with a frame invariant measure of the stress $f(\mathbf{T})$.

According to our assumptions, an elastic-type constitutive equation is valid at the moderate-stress regime

$$
\hat{\mathbf{T}}=\hat{\mathbf{T}}\left(\mathbf{B}_{e}\right), \quad \text { if } \quad f(\mathbf{T}) \leq \tau .
$$


where the stress in the current configuration $\mathbf{T}$ is obtained from $\hat{\mathbf{T}}$ by a push-forward: $\mathbf{T}=\hat{\mathbf{T}} \mathbf{F}_{p}^{T} / \operatorname{det}\left(\mathbf{F}_{p}\right)$. The tangential component of the stress vector relative to the surface identified by the normal $\mathbf{n}$ is

$$
\mathbf{t}(\mathbf{n})=\mathbf{T n}-(\mathbf{n} \cdot \mathbf{T n}) \mathbf{n}
$$

A suitable measure of the stress is

$$
f(\mathbf{T})=\max _{|\mathbf{n}|=1}|\mathbf{t}(\mathbf{n})|,
$$

representing the maximum shear stress occurring in the plane identified by the eigenvector corresponding to the maximum of $|\mathbf{t}(\mathbf{n})|$. It can be explicitly calculated that $f$ is half of the difference between the maximum and the minimum eigenvalue of $\mathbf{T}$.

When the tension overcomes the yield stress in terms of the stipulated measure $f$, energy is no longer elastically stored. The extra energy possibly provided by the work of external and internal (growth) forces is spent in unbinding at the cellular scale, i.e. material rearrangement at the macroscopic scale. Cells flow in mutual direction, dissipating energy, and determining an evolution of the intermediate configuration. In such regime the material behaves as a viscous fluid, with the theoretical complication that an amount of strain energy remains stored during the flow. Such a pictorial description is put into formal terms by the following constitutive equation

$$
\mathbf{L}_{p}=\frac{1}{2 \eta}\left[1-\frac{\tau}{f(\mathbf{T})}\right]_{+} \mathbf{F}_{e}^{T} \mathbf{T} \mathbf{F}_{e}^{-T}
$$

where the symbol $[\cdot]_{+}$denotes the positive part of the argument, $\eta$ is a dynamic viscosity. Neglecting the mappings by $\mathbf{F}_{e}$ and its inverse, due to the existence of an intermediate configuration, basically at the right hand side of Equation (8) there is the tensor deformation rate, i.e. the stress tensor that characterizes the viscous fluids. After some calculations, an evolution equation for $\mathbf{F}_{p}$ can be obtained. Therefore, in the present framework, (8) provides the evolution equation for the relaxed configuration.

The positive part function that appears at the right hand side of (8), depending on the value of the yield stress $\tau$, distinguishes between the elastic (reversible) behavior and the viscous (irreversible) behavior of cell aggregates. In Eq. (8), if the body undergoes a deformation corresponding to a stress below the yield stress, then the square parenthesis vanishes and $\mathbf{F}_{p}$ does not change; the natural configuration does not evolve and all the energy is stored elastically. If the measure of tension $f$ takes a value larger than the yield stress, then the reference configuration changes to release the stress in excess, until the yield condition defined by $f$ is reached again. An example of specific constitutive equation for this problem is reported in [15].

The complexity of the system of equations that model the growth of a solid tumor with non trivial mechanical behavior is currently treatable just in very simple geometries, when radial symmetry makes the problem onedimensional. In case of complex geometries, the complexity of the mathematical problem makes it attractive the idea to reconsider the model in terms of a cell-based point of view. In such an approach, single cells are individually discretized in their position and in their own interaction with the surrounding ones. The number of tumor cells in a multicell spheroid is large, but the number of degrees of freedom can be numerically afforded. The drawback of a cell-based approach is known: without the powerful tools of differential calculus, no predictions can be done about qualitative behavior and stability of the system. In a pure cell-based any the dependence of the solution on parameters can be just explored by sampling numerically the space of the parameters. The most convenient strategy (continuum vs. cellular) is still to be devised.

\section{VAsculogenesis}

When solid tumors reach a critical size, the nutrient uptake provided by mere diffusion becomes insufficient; the diffusion feeds just a rim of cells in the spheroid and the inner ones start dying in the core. At this stage the cells at the surface of the cluster start organizing a more efficient transportation system by creating an $a d$ hoc efficient network: a new vasculature.

The vasculogenesis in vitro is a biological system that effectively mimics such a behavior and represents a suitable 
playground to obtain a quantitative measure of the phenomena, as well to test methods aimed to inhibit its start up (anti-angiogenesis). When cultured in vitro in a suitable environment, endothelial cells are able to organize themselves to form capillary-like networks. Their apparent strategy has some peculiar features that can be experimentally explored, with the final aim to clarify the inner mechanisms and possibly transfer the relevant results to the angiogenetic phase of tumours, when new vessels are formed to offer a more efficient nutrient uptake to tumour cells.

The usual experimental setting is two-dimensional: a Petri dish is coated with matrigel, a surface that favors cell motility thanks to biochemical characteristics similar to those of living tissues. Human endothelial cells are dispersed in a physiological solution, which is poured on the top of the matrigel. After sedimentation due to gravity, cells move on the horizontal surface of the substratum giving rise to a process of aggregation and pattern formation. If the proper number of cells is used (cell density ranging from 100 to $400 \mathrm{cell} / \mathrm{mm} 2$ ), a vascular-like network develops. The entire process lasts 12-15 hours and evolves according to the following steps:

$\mathrm{i}$ in the first few hours (2-3 hours) cells migrate independently, keeping a round shape (Figures 2a-b). In this phase, cell motion is faster than later motion and shows a high persistence, that is a tendency to preserve their direction of movement.

ii the cells, attracted toward regions with higher cellular density, attach to the neighboring ones and, if possible, form a continuous multicellular network (Figure 2c);

iii the cells take a more elongated shape. Their motion becomes slow, they anchor themselves to the substrate, and, acting on the adhesion sites, start pulling the substratum and the other cells. The network slowly moves as a whole, undergoing a slow thinning process (Figure 2d), probably driven by the stress field generated by mutual tractions;

iv in the final phase, individual cells differentiate and fold up to form the lumen of the capillary, so that the formation of a capillary-like network takes place along the lines of the previously formed two-dimensional structure.

Two transitions are observed at low and high cell densities. The former is a percolative transition that occurs below the critical threshold of 100 cells $/ \mathrm{mm} 2$ and gives rise to groups of disconnected structures. This transition is analyzed in detail in [9]. The latter gives rise to thicker cords, and for very high cell density (say above 400 cells/mm2) to the formation of a continuous carpet of cells with holes or lacunae.

Mathematical literature on the subject is quite partitioned into two mainstreams, that in essence correspond to two different explanations for the basic mechanism of intercellular communication leading to pattern formation. The earlier thread is due to Murray, Oster, Manoussaki and coworkers $[12,13]$. They focus on the mechanical interactions between cells and extracellular matrix that occur when cells adhere to the underlying layer and stretch it. These authors observe that the tensional field caused by a cell pulling on the substratum creates a local indirect information about the concentration of the cells themselves on the surrounding surface. They therefore assume that cells directly detect the stress field of the layer and move on the surface along the tensional lines. Cells organization in soft media due to active mechanosensing has been confirmed by a number of experiments reported in the recent literature. As the tension depends on the number of pulling cells, the migrating ones move toward regions of larger cellular density. Qualitative analysis and numerical integration of the set of partial differential equations corresponding to the mathematical formalization of this idea demonstrate that network-like structures actually form on the basis of this assumption.

However, experimental works point out that, before mesenchymal motion activates, cells undergo a faster migration, driven by chemical factors such as Vascular Endothelial Growth Factor (VEGF). During this stage, a draft of the final network emerges, although it exhibits itself in the final form only after the stretching process. Even more important, laboratory investigations demonstrate that a characteristic mesh size independent of the number of cells exists, provided the latter are neither too many nor too few in order for the morphogenetic process to start up. The conjecture that the network forms just during the mesenchymal migration does not predict the existence of any characteristic length, so that the final mesh size would be dictated essentially by the initial conditions. 


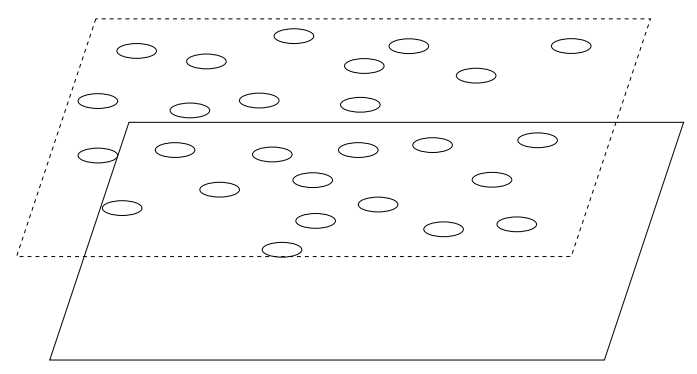

(a)

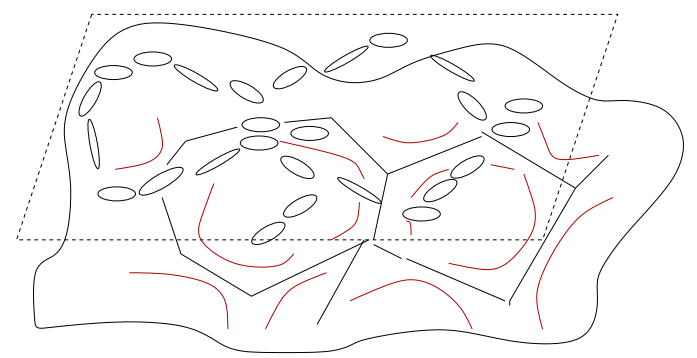

(c)

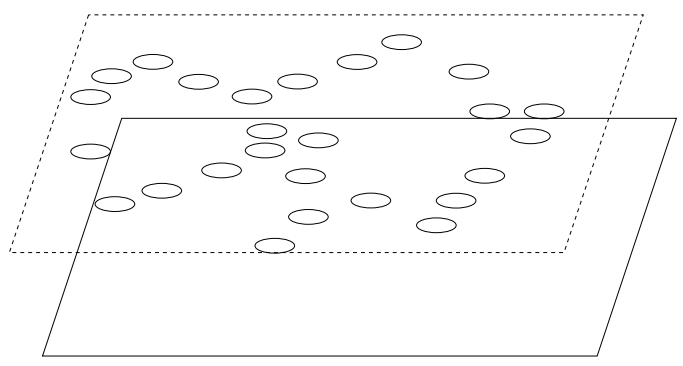

(b)

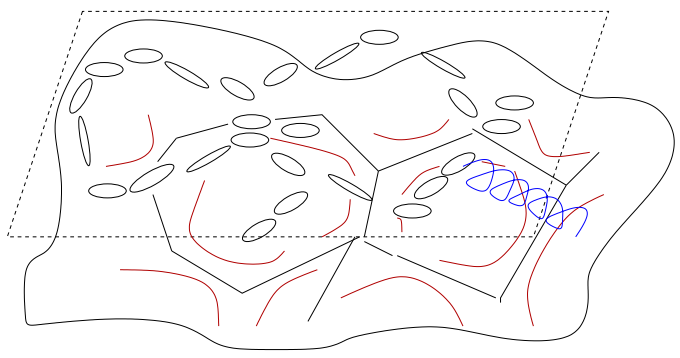

(d)

FiguRE 2. Random seeding of the cells (a), fast cell migration along the gradient of the chemoattractant concentration (b), traction between the cellular layer and the gel (c). In Figure (d) is pictorially shown the spring ideally connecting the two layers according to our model.

To obviate these difficulties of the theory, some authors propose mathematical models in which the chemotactic interaction is the real driver of network formation [9]. This assumption is supported by the observation that exogenous saturation of the signaling of some VEGF inhibits the networking. The mathematical model identifying the chemical signaling as the key mechanism of vasculogenesis predicts a characteristic length of the mesh size, which is in good agreement with the experiments.

The main drawback of a purely chemotactic model is that no steady state is asymptotically obtained: the solution blows up in a finite time [10]. A possible way to prevent this unsatisfactory prediction is to stabilize the system including the strain energy of the substratum, to be balanced by the chemotactic process. A unified view of the whole morphogenetic process of vascular networks, including both fast and slow migration is obtained if cells and substrate are described as a two-layers system, in which the vertical dimension can be neglected. The two layers are shallow, continuous, bodies that obey standard force balance equations including an interaction term, which couples the motion of the cells and the substratum. The balance of mass and force for the cell layer reads

$$
\begin{aligned}
\frac{\partial \rho}{\partial t}+\nabla \cdot(\rho \mathbf{v}) & =0 \\
\frac{\partial \rho \mathbf{v}}{\partial t}+\nabla \cdot(\rho \mathbf{v} \otimes \mathbf{v})+\nabla p(\rho) & =\rho \beta \nabla c+\gamma\left(\mathbf{u}-\mathbf{u}_{c}\right)
\end{aligned}
$$

where $\rho$ is the density of cells per unit volume, $\mathbf{v}$ their velocity, $p$ is the pressure due to the mutual interaction of the cells, $c$ is the density per unit volume of chemoattractant, $\mathbf{u}$ and $\mathbf{u}_{c}$ are the displacement of the substrate and the cells, respectively. 
The equations for the balance of force in the substrate and the reaction-diffusion of chemoattractant read:

$$
\begin{aligned}
-h \mu \Delta \mathbf{u}-h(\mu+\lambda) \nabla(\nabla \cdot \mathbf{u}) & =-\gamma\left(\mathbf{u}-\mathbf{u}_{c}\right) \\
-D \Delta c & =\alpha \rho-\tau^{-1} c .
\end{aligned}
$$

where $\dot{\mathbf{u}}_{c}=\mathbf{v}, \mu$ and $\lambda$ are the Lamé coefficients of the material in vertically averaged equations.

The equations are discretized by $256 \times 256$ nodes in a square domain with periodic boundary conditions. The elliptic equations (11-12) for the chemoattractant $c$ and for the matrigel displacement $\mathbf{u}$ are solved by a spectral method. The hyperbolic equations for mass conservation and momentum balance are solved by a finite volumes Godunov-type scheme. Chemotactic equations of hyperbolic type give rise to a number of intriguing questions in terms of its derivation from kinetic models [6] and in designing efficient numerical schemes [5].
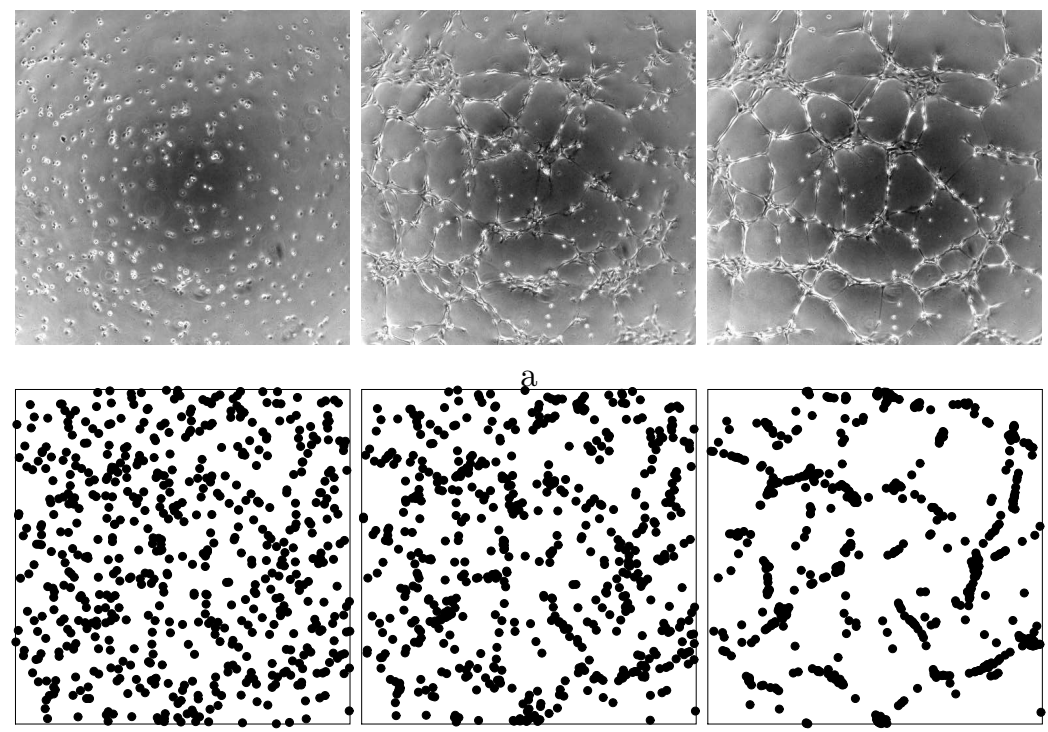

FiguRE 3. Early stages of the formation of capillary networks: comparison between the experimentally observed (a) and the numerically predicted (b) morphology. Here the black dots denote the position of material points of the material, initially randomly located and then transported by the velocity field.

The results are in good agreement with experiments, also from a quantitative point of view, in terms of statistically reproduced correlation. Notably, there is just one free parameters in the model. The Lamé constants $\lambda$ and $\mu$ are parameters of the matrigel material, the diffusion coefficient $D$ and the degradation and production rates $\tau, \alpha$ depend on the chemotactic factor (VEGF): all these quantities can be directly measured by independent experiments, at least in principle. It remains to be fixed the coefficient of the chemotactic force $\beta$, to be tuned in order to reproduce the correct time scale of the morphogenesis, and the free parameter $\gamma$.

The model adopted herein allows to include in the description both the (independent) reorganization of the cells according to the chemotactic signaling and the (coupled) stretching of the gel. This approach provides a satisfactory unified framework for the vasculogenesis process at all stages, and the numerical simulations suggest that a stable steady solution should exist in a physically significant range of parameters.

\section{FINAL REMARKS}

The two examples of tumor growth and the corresponding formal representation reported in this paper share one common characteristics: the mathematical model is based on conservation principles of mechanical 

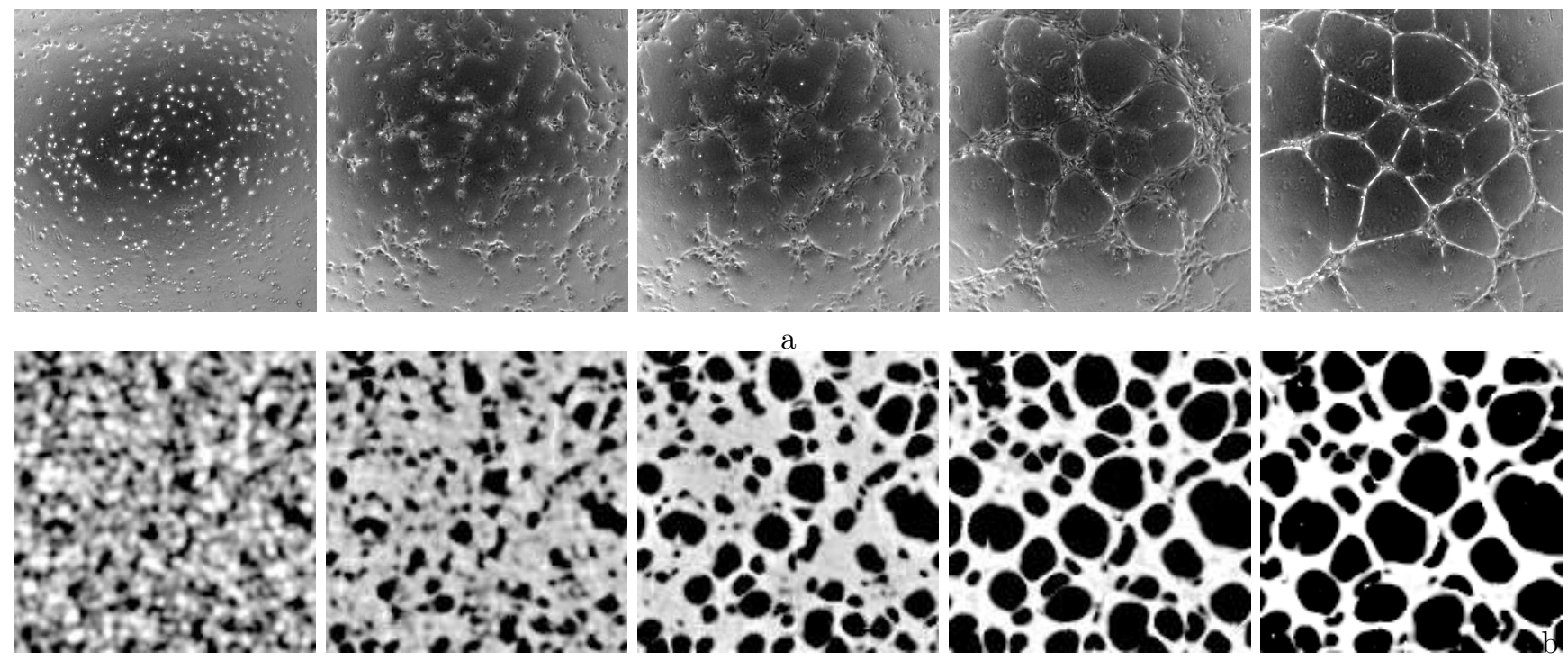

FiguRE 4. Formation of capillary networks: comparison between the experimentally observed (a) and the numerically predicted (b) morphology. In this graphical representation the cellular density is plotted in white if its value is above a given threshold.

quantities (mass, momentum). In both cases it appears that standard models are too poor, and some new ingredient is to be included in the description as suggested by the experimental observation. The non-standard component of the model is growth, for multicell spheroids, or the chemotactic force, in the case of vasculogenesis. This way to proceed has one main advantage, in my opinion. As far as as no new biophysical effects are included, one can straightfowardly inherit the general results in terms of well posedness, frame indifference, stability, that have been collected in the classical literature for inert matter. Then the new non-standard elements, like the chemotactic force appearing in equation (10) for instance, can be possibly stated in some known framework, or they simply offer new challenging mathematical questions.

Whatever the mathematical model adopted, the numerical simulation of solid tumor growth (in its avascular or vascular phase) is still in its infancy. One open question is the statement of the mathematical laws that rule the interplay between growth and mechanics; as discussed above, this aspect is less relevant than the biochemical factors, but not negligible. The interplay between nutrients, kinetics of growth factors and cell proliferation is much better understood, but the complexity of the such relations make quantitative predictions difficult, although recent examples exist [16]. Finally I want to mention that the application of the basic biological discoveries to medical applications faces a major difficulty in the uncertainty of parameter estimation and geometrical definition, that is completely unexplored from a mathematical point of view.

\section{REFERENCES}

[1] Ambrosi D and Preziosi L, On the closure of mass balance models of tumor growth, Math. Models Meth. Appl. Sciences, 12:737-754 (2002).

[2] Ambrosi D and Preziosi L, Cell adhesion mechanisms and stress relaxation in the mechanics of tumours, Biomechanics and Modeling in Mechanobiology, 8(5):397-413 (2009).

[3] Ambrosi D and Mollica F, The role of stress in the growth of a multicell spheroid, J. Math. Biol., 48(5):477-499 (2004).

[4] Byrne H, Modelling avascular tumour growth, in Cancer Modelling and Simulation, L. Preziosi ed., Chapman\&Hall/CRC, (2003).

[5] Filbet F and Shu CH, Approximation of hyperbolic models for chemosensitive movement, Siam J. Sci. Comput. 27(3):850872 (2005) 
[6] Filbet F, Laurencot F and Perthame B, Derivation of hyperbolic models for chemosensitive movement J. Math. Biol. 50(2):189207 (2005).

[7] Folkman J and Hochbergand M, Self-regulation of growth in three dimensions, J. Exp. Med., 138:745-753 (1973).

[8] Forgacs G and Foty RA and Shafrir Y and Steinberg MS, Viscoelastic properties of living embryonic tissues: a quantitative study, Biophys. J., 74(5):2227-2234 (1998).

[9] Gamba A, Ambrosi D, Coniglio A, de Candia A, Di Talia S, Giraudo E, Serini G, Preziosi L and Bussolino F. Percolation, morphogenesis and Burgers dynamics in blood vessels formation, Phys. Rev. Lett. 90, 11810/1-4 (2003).

[10] Kowalczyk R, Preventing Blow-up in a Chemotaxis Model. J. Math. Anal. Appl. 305:566-588 (2005).

[11] Helmlinger G, Netti PA, Lichtenbeld HC, Melder RJ and Jain RK, Solid stress inhibits the growth of multicellular tumour spheroids, Nature Biotech. 15:778-783 (1997).

[12] Manoussaki D, Lubkin SR, Vernon RB and Murray JD A mechanical model for the formation of vascular networks in vitro, Acta Biotheoretica 44:271-282 (1996).

[13] Murray JD and Oster GF, Cell traction models for generation of pattern and form in morphogenesis, J. Math. Biol. 19:265-279 (1984).

[14] Namy P, Ohayon J and Traqui P, Critical conditions for pattern formation and in vitro tubulogenesis driven by cellular traction fields, J. Theor. Biol. 227:103-120 (2004).

[15] Preziosi L, Ambrosi D and Verdier C, An Elasto-visco-plastic Model of Cell Aggregates, J. Theor. Biol., 262:35-47 (2010).

[16] Ribba B, Saut O, Colin T, Bresch D, Grenier E and Boissel JP, A multiscale mathematical model of avascular tumor growth to investigate the therapeutic benefit of anti-invasive agents, J. Theor. Biol., 243(4):532-541 (2006).

[17] Taber L, Towards a unified theory for morphomechanics, Transact A Math Phys Eng Sci., 367(1902):3555-3583 (2009).

[18] Tosin A, Ambrosi D and Preziosi L, Mechanics and Chemotaxis in the Morphogenesis of Vascular Networks, Bulletin of Mathematical Biology 68(7):1819-1836 (2006). 